\title{
Renal calcification in hypoparathyroid patients treated with calcium and vitamin D: Can biochemistry help?
}

Pathmanathan Sivatharshya ${ }^{1}$, Scott Tolhurst ${ }^{2}$, Emma Illingworth ${ }^{1}$, Claire Higham ${ }^{1}$, Peter J Trainer ${ }^{1}$, Phillip J Monaghan²

Hospital ${ }^{1}$ Department of Endocrinology, The Christie NHS Foundation Trust. ${ }^{2}$ The Christie Pathology Partnership, The Christie NHS Foundation Trust, Manchester M20 4BX, UK.

\section{Objectives}

1. To assess whether serum and urine biochemical parameters are associated with the presence of renal calcification in hypoparathyroid patients on Alfacalcidol therapy.

1. To establish if the spot urine calcium:creatinine method can be adopted as the main biochemical urine prognostic and monitoring test method.

\section{Methods}

Approval for all ethical and health and safety issues in the project was obtained from The Christie Trust clinical audit committee.(Reference CE14/1353)

The patients diagnosed with hypoparathyroidism or primary hypoparathyroidism and had received Alfacalcidol (Vitamin D) replacement therapy and receiving care in the Endocrine department of the Christie Hospital were included in the study.

The study period focused on was a 12 month audit of the laboratory database of paired requests for $24-\mathrm{h}$ urine calcium $(24 \mathrm{~h}-\mathrm{Ca})$, spot calcium:creatinine ratio (Ca:Creat), serum calcium, phosphate, urea and creatinine.

A review of case notes was performed to confirm aetiology of hypoparathyroidism, Alfacalcidol dose, duration of therapy and results of renal ultrasound scan (USS).

\section{Results}

A total of 34 patients were identified as having hypoparathyroidism and receiving Alfacalcidol therapy.

24h-Ca and Ca:Creat were notnormally distributed, however significant associations were found between $24 \mathrm{~h}-\mathrm{Ca}$ and $\mathrm{Ca}$ :Creat when log-transformed (linear regression bcoefficient $=0.64 ; 95 \% \mathrm{Cl} 0.36$ to 0.92 ; $p<0.001, \beta=0.63$ ).

17 patients had documented hypercalciuria evidenced by elevated 24h-Ca (5 patients), Ca:Creat (8 patients) or both (4 patients).

13 patients had undergone renal USS; 4 had evidence of renal calcification. Interestingly, these 4 patients each had an elevated $\mathrm{Ca}$ :Creat, in contrast with only one patient having elevated $24 \mathrm{~h}$ Ca.

No patient had hypercalcaemia. However, 20 patients had low, or lownormal serum adjusted calcium ( $\mathrm{Ca}<$ $2.2 \mathrm{mmol} / \mathrm{L}$ ); 9 of these patients having documented hypercalciuria evidenced by an elevated $24 \mathrm{~h}-\mathrm{Ca}$ ( $78 \%$ of patients) or Ca:Creat ( $89 \%$ of patients).

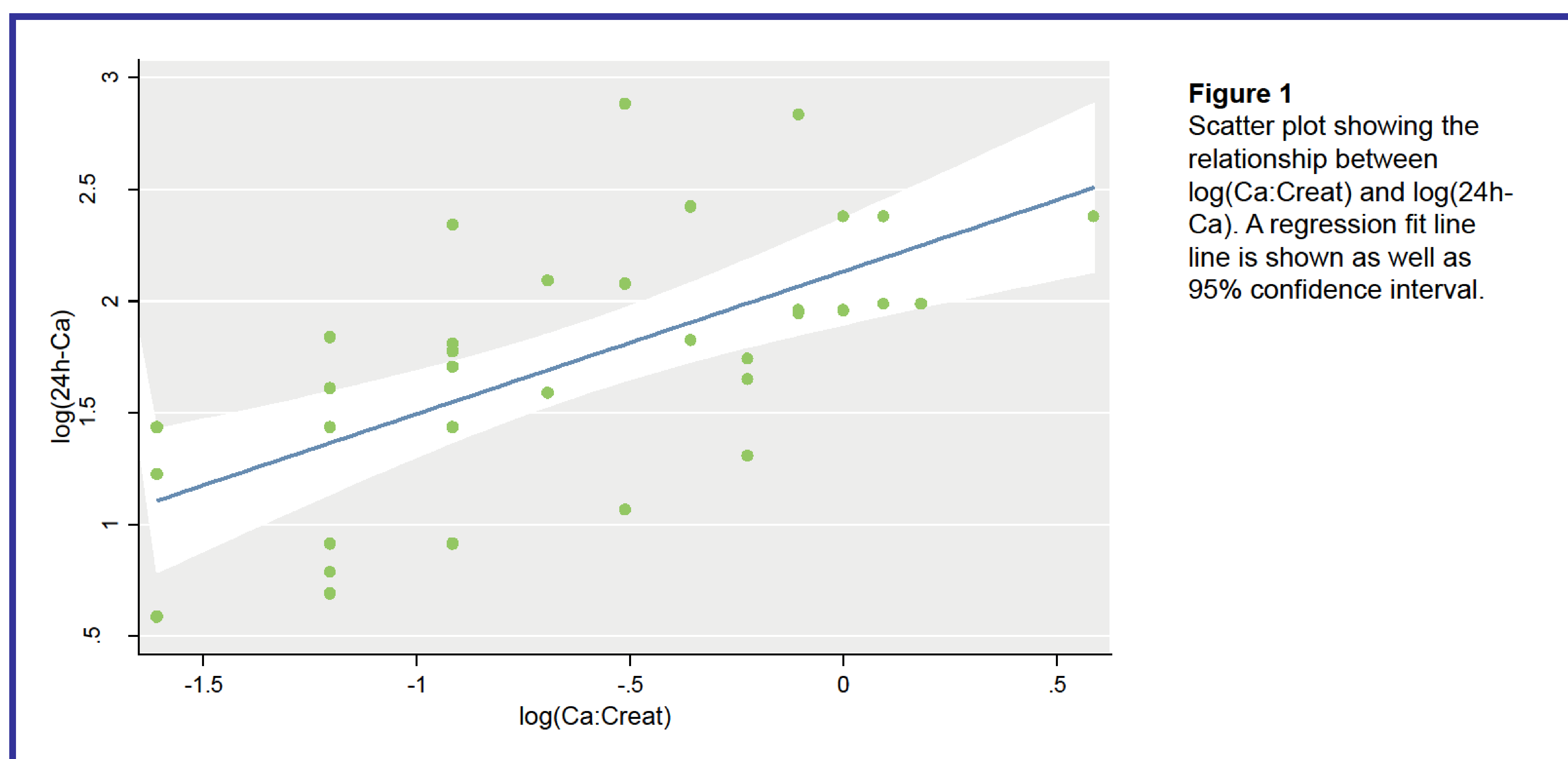

\section{Conclusions}

Ca:Creat ratio appears a sensible and convenient marker for the follow-up of patients on long term Alfacalcidol therapy to determine associated risk of renal calcification.

\section{References}

1. Gokce C Gokce O Baydinc C et al (1991). Use of random urine samples to estimate total urinary calcium and phosphate excretion. Arch Intern Med,151,pp.1587-1588

2. Hong, Y., Dublin, N., Razack, A., Mohd, M. and Husain, R. (2010). Twenty-four Hour and Spot Urine Metabolic Evaluations: Correlations Versus Agreements. Urology, 75(6), pp.1294-1298.

3. Jones, A. and Hansen, K. (2010). A Moratorium on the Spot Urine Calcium-to-Creatinine Ratio. Urology, 76(4), pp. 1021-1022. 